\title{
Genome analysis of Agrobacterium tumefaciens: Linkage map and genetic features of the left region of the linear chromosome
}

\author{
Devika M. De Costa, Katsunori Suzuki, Megumi Satou and Kazuo Yoshida* \\ Department of Biological Science, Graduate School of Science, Hiroshima University, \\ Higashi-Hiroshima 739-8526, Japan.
}

(Received 2 July 2001, accepted 9 November 2001)

\begin{abstract}
In addition to a unique tumor-inducing (Ti) plasmid, the plant pathogenic bacterium Agrobacterium tumefaciens has an unconventional chromosomal organization. Our previous studies on $A$. tumefaciens MAFF301001 revealed that it possesses a 2 $\mathrm{Mb}$ linear and a $2.8 \mathrm{Mb}$ circular chromosome plus a $206.479 \mathrm{kbp}$ Ti plasmid (pTiSAKURA). In this study, a linkage map for the left half of its linear chromosome covering a $900 \mathrm{kbp}$ region was constructed and the number of potential genes existing in the region was estimated. The linkage map consists of $31 \mathrm{BAC}$ and 8 lambda phage recombinants without any gaps. It confirmed the size and all the structural landmarks indicated in the corresponding region of our previously constructed physical map for the linear chromosome. Sequencing analysis of the end-regions of each linking clone led to the identification of 6 genes and another 27 potential genes or ORFs, including genes and/or gene clusters responsible for homologous recombination $(r u v B)$, trehalose/maltose sugar transport $(t h u R, t h u G)$ and alanine catabolism $(\mathrm{dadR})$. Two virulence-related gene homologues (attK and celB), previously reported in the circular chromosome of a different strain of A. tumefaciens were found in this region. These findings will provide a ready-to-use linkage map for further functional analysis of the linear chromosome.
\end{abstract}

\section{INRODUCTION}

A. tumefaciens, the bacterium causing crown gall disease in a wide range of dicot plants, is a subject of widespread research interest in the fields of plant molecular biology and plant pathology. Over the last few decades, detailed studies have been done to elucidate the nature of its pathogenicity (Zambryski, 1992; Hooykass and Beijersbergen, 1994; Sheng and Citovsky, 1996) and to develop its use as a tool in plant biotechnology (Klee et al., 1987). Besides pathogenic gene transfer between kingdoms, we also found trans-kingdom conjugative characteristics in A. tumefaciens (Nishikawa et al., 1992, 1998; Sawasaki et al., 1996).

In addition to the above-mentioned phytopathological and biotechnological importance, it has recently been reported that $A$. tumefaciens has an unusual genomic make-up in that it harbors one circular and one linear chromosome together with a giant Ti plasmid (AllardetServent et al., 1993; Suzuki et al., 2001). Fig. 1 shows the three genomic DNA molecules maintained at an

Edited by Hideo Shinagawa

* Corresponding author. E-mail: kyoshida@sci.hiroshima-u.ac.jp equimolar ratio in A. tumefaciens MAFF301001 strain (Suzuki et al., 2001). Most prokaryotes have circular chromosomes, while some possess linear ones, but the coexistence of a circular and a linear chromosome in $A$. tumefaciens is unique among the genome compositions known at this time (Casjens, 1998; Volf and Altenbuchner, 2000).

In A. tumefaciens, much of the research attention so far has been focused on Ti plasmid, as it is the genomic component which is mainly responsible for pathogenicity and T-DNA transfer. However, some involvement of $A$. tumefaciens chromosomal genes in the course of pathogenicity has been demonstrated. Most of these genes have been mapped on the circular chromosome (Douglas et al., 1985; Cooley et al., 1991; Winans, 1992; Suzuki et al., 2001) and a few on the linear chromosome (see Fig. 1(a) and (b), Suzuki et al., 2001). Additionally, there are reports that the linear chromosome is not homologous with the circular one (Allardet- Servent et al., 1993; Suzuki et al., 2001). So far, very little information is available about the gene organization of the linear chromosome. Therefore, it would be of interest to determine the functional consequences of having two topologically different chromosomes and the gene distribution in the two 
(a)

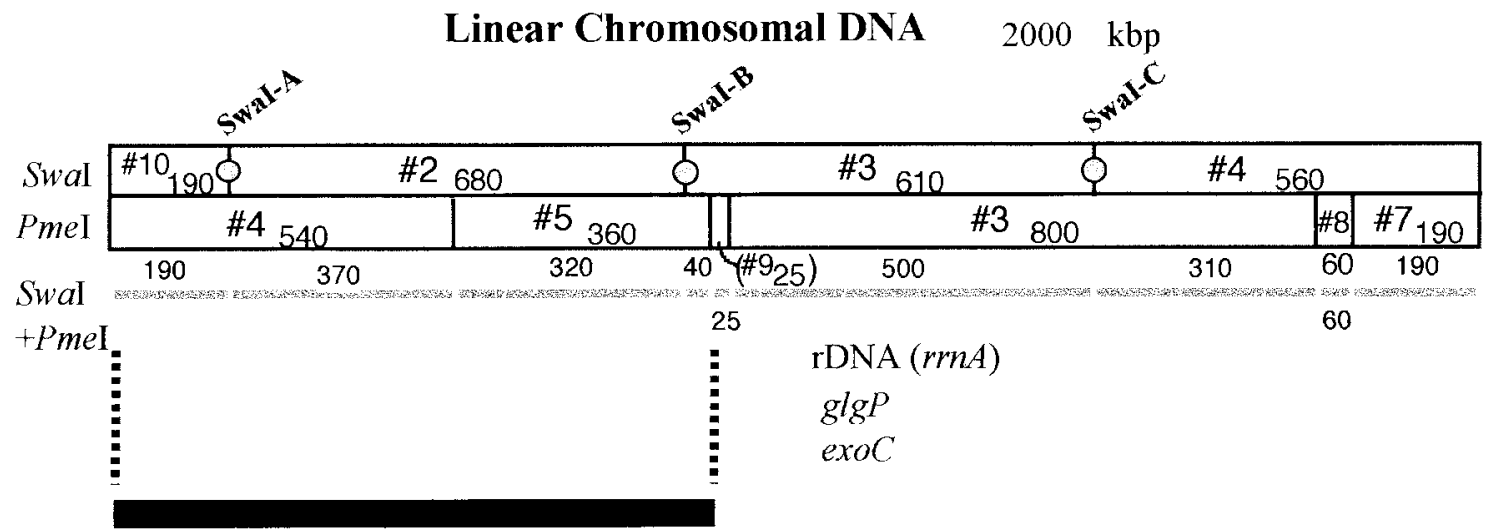

\section{Analyzed region in this study}

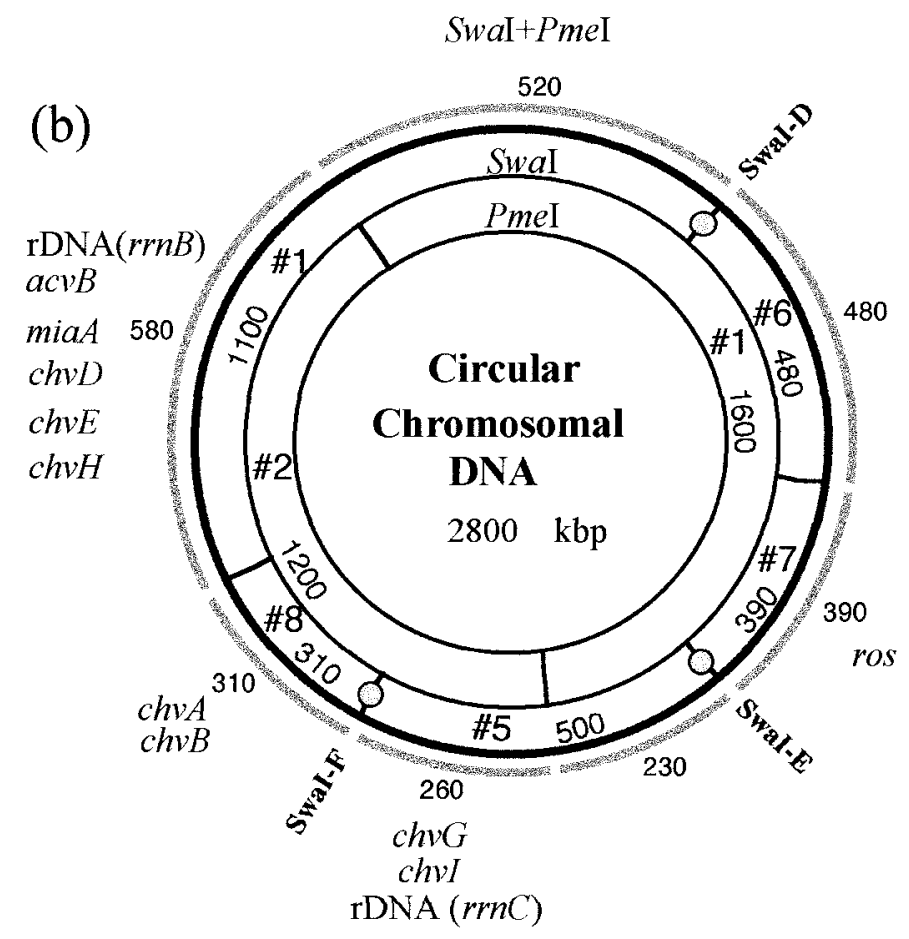

(c)

virA, virB1B2B3B4B5B6B7B8B9B10B11

VirG, virClC2,virDID2D3D4,virH, virE1E2,

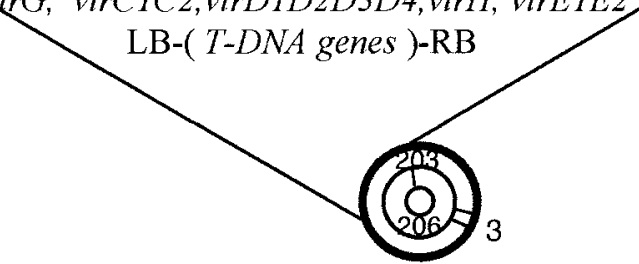

pTi-SAKURA plasmid $206.479 \mathrm{kbp}$

Fig. 1. The genome organization of A. tumefaciens MAFF301001. Physical and genetic maps indicate the locations of rDNA genes and genes responsible for virulence on the (a) linear chromosome of $2000 \mathrm{kbp}$, (b) circular chromosome of $2800 \mathrm{kbp}$ and (c) Ti plasmid (pTiSAKURA) of $206.479 \mathrm{kbp}$, which has been completely sequenced (Suzuki et al., 2000). SwaI and PmeI indicate the restriction enzymes used for physical map construction (see Suzuki et al., 2001). The symbol (\#) with numbers are serial names of the fragments based on PFGE separation and the values just below each fragment indicate their size in kbp. The thick horizontal line indicates the region which was analyzed in this study.

chromosomes. Furthermore, the left region of the linear chromosome is the most obscure area in which no gene has been mapped so far.

To address the above issues, it is our long-term intention to elucidate the structural and functional genomics of this bacterium. To this end, background information has already been obtained in our laboratory by complete sequencing of a typical nopaline type Ti-plasmid, pTiSAKURA (see Fig. 1(c), Suzuki et al., 2000; Hattori et al., 2000) and by constructing the physical maps for the linear and circular chromosomes of A. tumefaciens MAFF301001 (Suzuki et al., 2001). 
As shown by Redenbach et al. (1996) in the analysis of Streptomyces chromosome, construction of an ordered clone map is one of the powerful methods to overview bacterial chromosomes genetically. Among the vectors used for constructing gene libraries, bacterial artificial chromosomes (BACs)/fosmids can maintain insert DNA most stably because of its low copy number (Kim et al., 1992, 1996). Failure to cover the whole genome in Streptomyces (Redenbach et al., 1996) and in Mycobacterium tuberculosis (Wlofgang et al., 1996) even after screening of a large number of clones show the necessity of an additional DNA bank in a different type vector.

The present study was conducted to characterize the structural and functional features of the linear chromosome of A. tumefaciens MAFF301001. Here we report the construction of a linkage map and the genetic features of the left half of the linear chromosome. This is the first report to give such information, which will be indispensable for identifying important physical and genetic landmarks of the linear chromosome of A. tumefaciens.

\section{MATERIALS AND METHODS}

Bacteria, vectors and culture conditions. $A$. tumefaciens MAFF301001 which harbors a nopaline type Ti plasmid (pTi-SAKURA) (Suzuki et al., 2000) was the source of DNA throughout this study. BAC vectors (fosmids), pFOS1 (Kim et al., 1992) and pBeloBAC11 (Kim et al., 1996) as well as a lambda phage vector, DASHII (Stratagene, CA, USA) were used as cloning vectors. $E$. coli SURE was used as the cloning host for pFOS1 and pBeloBAC11 transformants. Lambda phage recombinants were cloned in $E$. coli XL1- Blue MRA (P2) strain. Agrobacterium was cultured in $\mathrm{YEM}+\mathrm{Mg}^{2+}$ medium at $28^{\circ} \mathrm{C}$ with vigorous shaking. LB broth and medium supplemented with chloramphenicol $(15 \mu \mathrm{g} / \mathrm{ml})$ were used for cultivating $\mathrm{BAC}$ recombinants at $37^{\circ} \mathrm{C}$. Lambda phage recombinants were cultivated in LB agar and NZYM broth.

Preparation of DNA. Total DNA of A. tumefaciens was isolated with extreme care to minimize physical shearing from $100 \mathrm{ml}$ of liquid culture as described by Suzuki et al. (2001). The plasmid DNA of BAC recombinants was isolated by the alkaline-SDS miniprep method as well as by $\mathrm{CsCl}$ gradient ultracentrifugation (Sambrook et al., 1989). Lambda DNA isolation was done according to the method described in Suzuki et al. (1998).

Construction of genomic DNA libraries. Three different genomic libraries of $A$. tumefaciens were constructed in pFOS1, pBeloBAC11 and DASHII vectors. To construct the pFOS1 library, total Agrobacterium DNA was digested with $M b o I$ enzyme to obtain $40 \mathrm{~kb}$ DNA fragments and the DNA was dephosphorylated subsequently.
pFOS1 plasmid DNA was completely digested with AatII, dephosphorylated and then cleaved with BamHI. Subsequently, MboI-digested Agrobacterium DNA was ligated with a 10 -fold molar excess of pFOS1 DNA. The ligated product was packaged in vitro using a Lambda Inn Kit (Nippon Gene, Japan) and the recombinants were selected on LB agar containing chloramphenicol (15 $\mu \mathrm{g} /$ $\mathrm{ml})$.

The pBeloBAC11 library contained total Agrobacterium DNA that was partially digested with HindIII and used for further scaffolding of the chromosomal region. The DASHII library used for gap filling contained insert DNA which was partially digested with $\mathrm{MboI}$ and subsequently ligated into BamHI-cleaved vector.

Sub-library separation. Colony blots of pFOS1 and pBeloBAC11 libraries were hybridized with probes prepared from four fragments of the linear chromosome (i.e. PmeI-\#4, PmeI-\#5, SwaI-\#2 and SwaI-\#10) separated by PFGE (pulsed field gel electrophoresis) according to the macro-restriction map of the linear chromosome (see Fig. 1(a)).

Preparation of probes and filters and hybridization techniques. End-specific RNA probes were prepared by in vitro transcription using the T7, SP6 and T3 promoters of vectors. BAC recombinant DNA was digested with a suitable restriction enzyme prior to end-specific probe preparation to obtain a reasonable length of insert DNA without destroying the promoter sites. pFOS1 recombinants were digested with EcoRI whereas pBeloBAC11 and DASHII clones were digested with either EcoRV or BanIII. The digested DNA was subjected to RNA polymerase reaction with Dig-11-UTP RNA labeling mix (Roche, Germany).

BAC clones stored in high density micro-well plates were replicated using a pin replicating device onto Hybond-N (Amersham Pharmacia Biotech, U.K.) filters. Filter membranes of lambda clones were prepared as described by Sambrook et al. (1989). Southern blots on Hybond-N filters were prepared using a commercial vacuum blotter. All the pre-hybridization, washing and developing conditions were according to the manufacture's instruction manual (Boehringer Mannheim, Germany/ Roche, Germany). Autoradiograms were analyzed by a BAS image analysis system (FUJI Photo Co. Ltd., Japan).

Assembly of contigs. Contigs were assembled by hybridizing PmeI-\#4 and PmeI-\#5 sub-libraries with T7 and SP6 end-specific RNA probes of the BAC recombinants. Firstly, several clones belonging to a sub-library were randomly selected as starting points for walking. Extension of contigs was performed by successive hybridizations. Clones detected by end-specific probes were verified by analyzing their restriction fragment profiles (Coulson and 
Sulston, 1990). Comparison of restriction profiles was also used to determine the clones that were aligned distantly to the probe-clone itself and one such clone was selected for the next round of hybridization as described by Suzuki et al. (1998). Hybridization results were counterchecked by comparing the results given by overlapping end-specific probes at each linking clone of the contig. Moreover, overlaps were confirmed by constructing EcoRI physical maps for the overlapping clones in contigs by using a Cos-Plan Kit (Nippon Gene, Japan) according to the method described by Rackwitz et al. (1985).

Sequencing and homology search. Nucleotide sequences at two ends of insert DNA of the linking clones were determined by using universal T7, SP6 and T3 primers. Sequencing was performed by using Dye deoxy terminator sequencing kits and automatic DNA sequencer models 373A and 310 (Perkin-Elmer, USA). DNA/amino acid homology searches were carried out by using the FASTA/DDBJ program. End-regions which showed higher homologies with the FASTA search were extended to obtain ORFs by the primer walking method (Hattori et al., 1997) and intact fosmid and lambda recombinants were used as the source of template DNA.

Accession Numbers. End-region sequences of 39 clones and extended nucleotide sequences of some regions belonging to the linkage map of the left region of the linear chromosome have been deposited in DDBJ/EMBL/GenBank databases under the accession numbers ranging from AB072619 to AB072696 (Table 2).

\section{RESULTS AND DISCUSSION}

Genomic libraries. As shown in Table 1, we constructed genomic libraries of $A$. tumefaciens MAFF301001 by using three types of vectors, namely, pFOS1, pBeloBAC11 and DASHII. The pFOS1 library, which was prepared initially, can cover 4 times the whole bacterial genome. Further scaffolding of the linear chromosome was done with the pBeloBAC11 library, which has 6.7 genomic equivalents. The DASHII library was used mainly for filling gaps, and corresponds to 6.6 genomic equivalents. According to the equation of Clarke and Carbon (1976), any gene of interest could be found in the pFOS1 library with $95 \%$ probability, while the corresponding probability value for the pBeloBAC11 and DASHII libraries was $99 \%$.

Assembly of contigs. Accurate contig assembly is a salient step in linkage map construction. As the first step of this process, sub libraries for PmeI-\#4 and PmeI-\#5 regions were separated from the $\mathrm{BAC}$ genomic libraries based on the physical map of the linear chromosome (see Fig. 1(a) and Fig. 2(a) (b), Suzuki et al., 2001). Clones belonging to these sub libraries were used to assemble contigs for the left region of the linear chromosome. According to hybridization results of the end-specific RNA probes, clones of these BAC sub libraries were assembled into 4 contigs (Fig. 2(c), De Costa et al., 2000). These 4 BAC contigs, Contig I, II, III and IV were positioned and oriented based on the following information.

During the process of contig formation, the locations and directions of the contigs were determined by comparing PmeI-\#4 and PmeI-\#5 sub library clones with the clones detected by each end-specific hybridization. This helped for determining the position of the contigs as well as for detecting any false-positive hybridizations. Digestion of recombinant DNA by $S w a I$ and/or PmeI enzymes detected the presence of $S w a \mathrm{I}$ and $P m e I$ restriction sites in $1 \mathrm{P} 15$, LM3, LJ3 and LH9 clones. The results confirmed the presence of SwaI restriction site(s) in the clones1P15, LM3 and PmeI restriction site(s) in the clones LJ3, LH9, respectively (Fig. 2 (c)). These data verified the orientation of the contigs according to the physical map of the linear chromosome. Moreover, clones detected when the junction clone SwaI-A (see Fig. 1(a), Suzuki et al., 2001) was used as a probe were also used to determine the orientation of Contig I, which is at the extreme left end. By this

Table 1. Total genomic libraries used and their relevance to linkage map of the left region of the linear chromosome

\begin{tabular}{c|c|c|c|c}
\hline \hline $\begin{array}{c}\text { Vectors used for } \\
\text { libraries }\end{array}$ & $\begin{array}{c}\text { Total no. of } \\
\text { clones in library }\end{array}$ & $\begin{array}{c}\text { Average insert } \\
\text { size of a clone } \\
\text { (mean of 20) } \\
(\mathrm{kbp})\end{array}$ & $\begin{array}{c}\text { No. of clones in } \\
\text { the linkage map }\end{array}$ & $\begin{array}{c}\text { Length covered } \\
\text { by each type of } \\
\text { vector in the } \\
\text { linkage map } \\
\text { (kbp) }\end{array}$ \\
\hline pFOS1 & 549 & 38 & 15 & 570 \\
pBeloBAC11 & 786 & 45 & 16 & 720 \\
DASHII & 1920 & 8 & 144 \\
\hline \multicolumn{2}{l}{ Length covered by the total number of linking clones (kbp) } & & 1434 \\
Length of the left region of the chromosome (kbp) \\
\% overlap
\end{tabular}

* According to the physical map of the linear chromosome (Suzuki et al., 2001) 


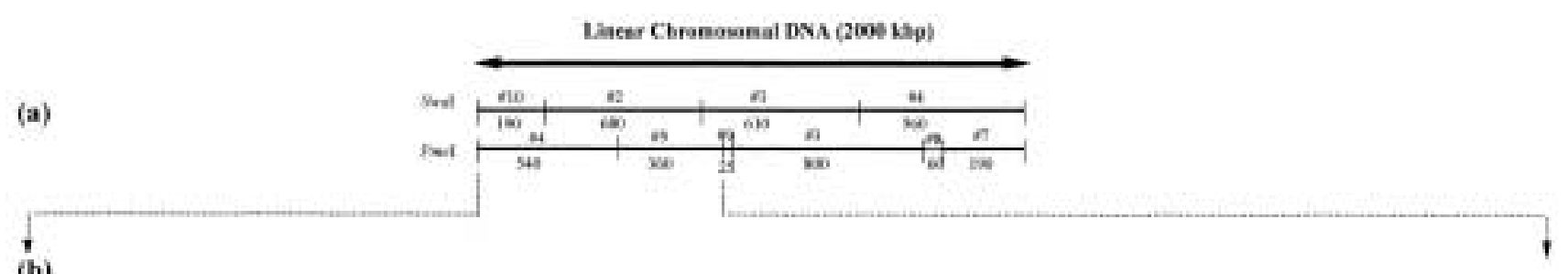

(b)
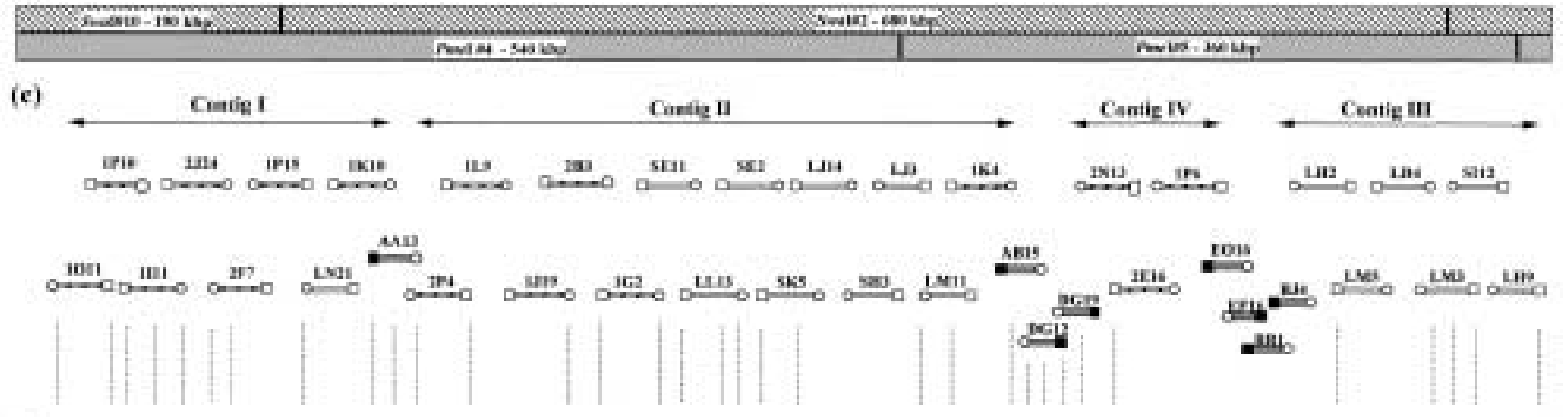

(d)

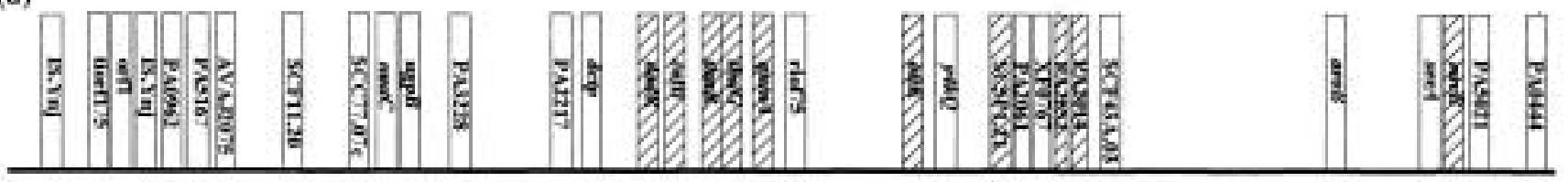

Fig. 2. Linkage map of the linear chromosome of A. tumefaciens MAFF301001. (a) Physical map of the linear chromosome (see Fig. 1 (a)). (b) Structural details of the left region of the linear chromosome. The upper hatched bar indicates the SwaI restriction map and the lower shaded bar indicates the PmeI restriction map, including the sizes of each fragment. Vertical lines are the locations of restriction sites (Suzuki et al., 2001). (c) Linkage map of fosmid and lambda clones. Symbols for the linkage map are as follows: open bars = pFOS1 clones, hatched bars = pBeloBAC11 clones, dotted bars = DASHII clones, circles = T7 ends, open squares = SP6 ends, solid squares $=\mathrm{T} 3$ ends. Clones just below the vertical lines of $(\mathrm{b})$ contain SwaI or PmeI restriction sites. Clones were designated according to their positions in high-density microtiter plates during the genomic library preparation; the three-character code indicates the plate, row and column, respectively. Contigs I, II, III and IV indicate the BAC contigs assembled initially. The numbering of these contigs is according to the order of assembly. (d) Localization of estimated genes in the left region of the linear chromosome. The gene/ORF name given for the best match by the FASTA DNA homology search is indicated in each box (see Table 2). Hatched boxes indicate the regions selected for sequencing extension. Dotted vertical lines between (c) and (d) indicate the end-region responsible for a particular homologue. Diagrams are not according to an exact scale.

method, the position and orientation of Contigs I, II and III were determined. Contig IV belonged to the PmeI-\#5 macro-restriction fragment with a known location but not having a known orientation.

The gaps between these 4 BAC contigs were filled by using a genomic library prepared in lambda phage (DASHII) vector. Southern hybridization of genomic DNA with BAC contig-end probes revealed a gap of approximately $10 \mathrm{kbp}$ between Contigs I and II (data not shown). Similarly, approximately $20 \mathrm{kbp}$ gaps were found between Contigs II and IV as well as between Contigs IV and III. Chromosomal walking in the DASHII library completely filled in the three gaps that existed between the BAC contigs. A single lambda clone was sufficient to fill the gap between Contigs I and II. Three lambda clones filled the gap between Contigs II and IV and four such clones were sufficient to fill the gap between Contigs IV and III (Fig. 2 (c)).

The linkage results were further confirmed by compar- ing $E c o$ RI restriction profiles of the set of clones detected by each end-specific probe.

Linkage map. Thus, assembly of BAC contigs and subsequent gap filling by use of DASHII clones led us to construct the final linkage map for the left region of the linear chromosome (Fig. 2 (c)). This complete linkage map consists of at least the overlapping clones of 31 BACs and 8 lambda phages without any gaps. The left region of the linear chromosome, covering PmeI-\#4 and PmeI-\#5 macro-restriction fragments, is approximately $900 \mathrm{kbp}$ in length (Suzuki et al., 2001). It is $45 \%$ of the length of the whole linear chromosome and $18 \%$ of the total genome of A. tumefaciens. The estimated length that can be covered by the total overlapping clones in the linkage map is 1434 kbp (Table 1). Hence, the overlapping clones in this linkage map are sufficient to cover the entire left region of the linear chromosome (except the extreme left end of the linear chromosome), with $37 \%$ overlap/clone. 
Gene estimation. The end-regions of all the clones in the linkage map were partially sequenced. Upon editing by using the SeqED program (Perkin Elmer-ABI), the resultant DNA sequences were subjected to a DNA/amino acid homology search. Table 2 shows the DNA homologies for all the end-regions which showed more than 200 Z-score values by FASTA search. These data were compared with the databases and based on these information, for each match the probable function and direction of transcription were deduced. The amino acid homologies deduced for them were identical to the deduced DNA homologies. Genes identified in the left region of the lin- ear chromosome by homology search are shown in Fig. 2 (d). According to the available data, the gene frequency in this region was estimated to be $42 \%$ (best hits/sequenced ends).

The average end region length which could be read by a single sequencing attempt was $400 \mathrm{bp}$. Therefore, endregion sequencing has covered approximately a $30 \mathrm{kbp}$ (sequenced length times number of contig ends) region along the linkage map.

A homology search revealed a broad spectrum of interesting functional motifs in the left region of the linear chromosome (Table 2 and Fig. 2(d)). These include

Table 2. Homologies shown by end-region sequences of the linking clones in the left region of the linear chromosome.

\begin{tabular}{|c|c|c|c|c|c|}
\hline $\begin{array}{c}\text { Clone end } \\
\text { (Accession No.) }\end{array}$ & $\begin{array}{l}\text { Best match } \\
\text { gene/ORF }\end{array}$ & $\begin{array}{l}\text { Accession No./ } \\
\text { Organism }\end{array}$ & $\begin{array}{l}\text { Product/ } \\
\text { Function }\end{array}$ & $\begin{array}{l}\text { \% identity/ } \\
\text { Gene } \\
\text { covered(\#) }\end{array}$ & $\begin{array}{l}\text { Z- } \\
\text { score }\end{array}$ \\
\hline 1O11T7 (AB072619) & IS. Ynj & AF242881/Agrobacterium tumefaciens & similar to IS 1313 of pTiB 0542 & $72.0 / 31.5$ & 777 \\
\hline 1O11SP6 (AB072620) & tiorf175 & $\begin{array}{l}\text { AB016260/Agrobacterium tumefaciens } \\
\text { pTi- SAKURA }\end{array}$ & probable IS ORF1 & $99.6 / 48.7$ & 2376 \\
\hline 1I11SP6 (AB072623) & orf1 & U19149/Agrobacterium tumefaciens & IS1312 ORF1 pseudogene & $62.2 / 7.5$ & 240 \\
\hline 1P10T7 (AB072622) & IS. Ynj & AF242881/Agrobacterium tumefaciens & similar to IS 1313 of pTiB0542 & $66.7 / 47.2$ & 898 \\
\hline 1I11T7 (AB072624) & PA0962 & AE004530/Pseudomonas aeruginosa & probable DNA binding stress protein & $59.1 / 34.7$ & 214 \\
\hline 2F7T7 (AB072627) & PA5167 & AE004929/Pseudomonas aeruginosa & probable C4-dicarboxylate binding protein & $57.5 / 39.3$ & 363 \\
\hline 2J24T7 (AB072626) & HVAJ2075 & AJ002075/Haloferax volcanii & citrate synthase & $75.0 / 7.0$ & 253 \\
\hline *LN21T7 (AB072631) & SCF11.20 & AL132662/Streptomyces coelicolor & probable sugar transport inner membrane protein & $58.3 / 32.3$ & 285 \\
\hline *AA13T3 (AB072635) & $\mathrm{SCC} 77.07 \mathrm{c}$ & AL136503/Streptomyces coelicolor & putative carbohydrate kinase & $58.0 / 24$ & 244 \\
\hline *1K10T7 (AB072634) & $m n t C$ & AY010757/Neisseria gonorrhoeae & periplasmic binding protein & $57.8 / 33.0$ & 356 \\
\hline *AA13T7 (AB072636) & $u g p B$ & U00039/E.coli & periplasmic binding protein & $58.6 / 22.6$ & 286 \\
\hline *2P4SP6 (AB072638) & PA3228 & AE004745/Pseudomonas aeruginosa & probable ATP binding protein/ permease $\mathrm{ABC}$ transporter & $64.7 / 9.7$ & 233 \\
\hline 1J19T7 (AB072642) & PA2217 & AE004648/Pseudomonas aeruginosa & probable aldehyde dehydrogenase & $69.0 / 8.7$ & 277 \\
\hline 1G2SP6 (AB072645) & dctp & X63974/Rhodobacter capsulatus & periplasmic C4-dicarboxylate binding protein & $57.7 / 33.6$ & 301 \\
\hline 1G2T7 (AB072646) & $d a d R$ & AJ249196/Rhizobium leguminosarum bv.viciae & alanine catabolic operon regulator & $68.9 / 80.8$ & 891 \\
\hline LL13SP6 (AB072649) & celB & L38609/Agrobacterium tumefaceins & involved in cellulose synthase & $87.6 / 16.6$ & 1488 \\
\hline SE2SP6 (AB072651) & thuR & AF175299/Sinorhizobium meliloti & putative regulatory protein & $62.8 / 21.3$ & 362 \\
\hline LL13T7 (AB072650) & thuG & AF175299/Sinorhizobium meliloti & putative inner membrane permease & $80.4 / 47.4$ & 1296 \\
\hline SK5SP6 (AB072653) & $q u m A$ & AF109471/Xanthomonas campestris & pyrroloquinate-quinone-dependent quinate dehydrogenase & $71.4 / 17.1$ & 1014 \\
\hline *LJ14SP6 (AB072655) & riorf75 & AP002086/Agrobacterium rhizogenes & hypothetical $\mathrm{ABC}$ transporter & $66.8 / 15.4$ & 416 \\
\hline *LM11T7 (AB072661) & $a t t K$ & U59485/Agrobacterium tumefaciens & succinate-semialdehyde dehydrogenase & $64.8 / 35.7$ & 847 \\
\hline 1K4SP6 (AB072663) & $\mathrm{y} 4 \mathrm{kQ}$ & AE000082/Rhizobium spp. & hypothetical protein & $72.6 / 80.0$ & 827 \\
\hline *1K4T7 (AB072664) & SC5F1.21 & AL450165/Streptomyces coelicolor & putative oligopeptide $\mathrm{ABC}$ transport ATP binding protein & $69.1 / 28.5$ & 479 \\
\hline *DG12T7 (AB072667) & PA2061 & AE004632/Pseudomonas aeruginosa & probable ATP-binding component of ABC transporter & $86.7 / 4.0$ & 239 \\
\hline$* \mathrm{AB} 15 \mathrm{~T} 7$ (AB072666) & XF0767 & AE003917/Xylella fastidiosa & transcriptional regulator & $58.0 / 79.6$ & 278 \\
\hline DG12T3 (AB072668) & PA2552 & AE004683/Psedomonas aeruginosa & probable acyl-CoA dehydrogenase & $71.8 / 34.0$ & 893 \\
\hline$* 2 \mathrm{~N} 13 \mathrm{~T} 7(\mathrm{AB} 072671)$ & PA2014 & AE004627/Pseudomonas aeruginosa & probable enoyl-CoA carboxyl transferase beta chain & $66.0 / 26.4$ & 706 \\
\hline *2E16SP6 (AB072673) & SCF43A.03 & AL096837/Streptomyces coelicolor & probable oxydoreductase & $63.3 / 21.1$ & 641 \\
\hline *LM5SP6 (AB072687) & $\operatorname{aroB}$ & AJ002783/Nisseria gonorrhoeae & 3-dehydroquinate synthase & $65.0 / 25.8$ & 476 \\
\hline *LD4T7 (AB072690) & $\operatorname{ser} A$ & AL035569/Streptomyces coelicolor & D-3 phosphoglycerate dehydrogenase & $57.6 / 19.8$ & 287 \\
\hline *SI12T7 (AB072693) & $\operatorname{ruv} B$ & AF175525/Rhizobium etli & repair and recombination protein, Holliday junction & $80.0 / 49.5$ & 1403 \\
\hline & & & binding protein, Holliday branch migration protein & & \\
\hline *LM3SP6 (AB072692) & PA5021 & AE004915/Pseudomonas aeruginosa & probable sodium/Hydrogen antiporter & $62.2 / 16.6$ & 342 \\
\hline *LH9SP6 (AB072696) & PA0444 & AE004481/Pseudomonas aerugenosa & $\mathrm{N}$ - carbamyl-beta-alanine amidohydrolase & $56.7 / 23.1$ & 332 \\
\hline
\end{tabular}

(\#) The proportion of the nucleotide length of a query sequence to that of a subject gene which we used to calculate the \% identity as the best match gene in the database. An assumption was made that two genes are same in size.

(*) End-regions which show high homology levels with Mesorhizobium loti genome in addition to the best matching organisms given in the table.

Following sequences did not show counterpart homologues with Z scores higher than 200: 1P10SP6 (AB072621), 2J24SP6 (AB072625), 2F7SP6 (AB072628), 1P15T7 (AB072629), 1P15SP6 (AB072630), LN21SP6 (AB072632), 1K10SP6 (AB072633), 2P4T7 (AB072637),1L9SP6 (AB072639), 1L9T7 (AB072640), 1J19SP6 (AB072641), 2B3SP6 (AB072643), 2B3T7 (AB072644), SE11SP6 (AB072647), SE11T7 (AB072648), SE2T7 (AB072652), SK5T7 (AB072654), LJ14T7 (AB072656), SH3T7 (AB072657), SH3SP6 (AB072658), LJ3T7 (AB072659), AB15T3 (AB072665), DG19T7 (AB072669), DG19T3 (AB072670), 2N13SP6 (AB072672), 2E16T7 (AB072674), 1P6T7 (AB072675), 1P6SP6 (AB072676), EO16T3 (AB072677), EO16T7 (AB072678), EF16T7 (AB072679), EF16T3 (AB072680), BB1T3 (AB072681), BB1T7 (AB072682), BJ4T3 (AB072683), BJ4T7 (AB072684), LH2T7 (AB072685), LH2SP6 (AB072686), LM5T7 (AB072688), LD4SP6 (AB072689), LM3T7 (AB072691), SI12SP6 (AB072694), LH9T7 (AB072695) 
housekeeping, transport-system-related, virulence and mobile-function-related genes, as explained below. Several end-sequences showed high levels of homology with the genes of $A$. tumefaciens itself. The rest showed high homology with those of taxonomically closely related organisms (i.e. Agrobacterium rhizogenes, Sinorhizobium meliloti, Mesorhizobium loti and Rhizobium spp,) as well as with those of distantly related organisms. The latter are probably due to the conserved functional characteristics of those sequences even in taxonomically distant organisms.

Housekeeping gene-related homologues. It is interesting to note that several end-region sequences of this region of the chromosome showed high homology to genes with housekeeping functions, such as genes involved in homologous recombination, metabolic/catabolic pathways and ion channeling. The $r u v B$ gene found at the T7 end of clone SI12 (Table 2 and Fig. 2 (d)) is one of the components responsible for the late stages of homologous genetic recombination and the recombinational repair of damaged DNA (West, 1997). It shows $85 \%$ identity with the ruvB gene of Rhizobium etli. The nucleotide sequence of the T7 end of clone SI12 showed a wide range of homology level with related sequences from a broad spectrum of organisms.

As shown in Table 2, several other genes such as PA2014 (probable enoyl-CoA carboxyl transferase beta chain), PA2552 (probable acyl-CoA dehydrogenase), serA, $d a d R$, and XF0767 (probable transcriptional regulator) which are components of certain metabolic/catabolic pathways were also tentatively identified. The sodium/ hydrogen antiporter (PA5021) identified at LM3SP6 is an interesting gene homologue due to its involvement in survival in environments with high $\mathrm{pH}$ and salt concentrations (Vimont et al., 2000).

Transport system-related gene homologues. Ten end-region sequences in this area of the linear chromosome showed homology with components of different transport systems (Table 2). The regions encoding transport systems possess several components of those operons such as periplasmic proteins and membrane proteins (Ames, 1986). As the structural composition of transport systems are highly conserved among many other bacteria, the neighboring regions of these putative genes may encode the rest of the components of transport systems. Such a possibility has already been shown to be true in the end-regions of overlapping clones SE2SP6 and LL13T7, in which thuR and thuG homologues of Sinorhizobium meliloti are components of the operon of the trehalose/maltose transport system (Table 2). Completed ORFs present in the LL13T7 and SE2SP6 end regions show $86 \%$ and $64 \%$ amino acid homology to the thuG and thuR genes respectively, in Sinorhizobium meliloti, which is taxonomically close to Agrobacterium. Moreover, further analysis of a greater extent of this region showed homology to the thuF gene of the same operon, which suggests that indeed extensive operons may exist in this region.

Virulence-related gene homologues. Four end-regions, namely, LM11T7, LL13SP6, SK5SP6 and LM5SP6, showing homology with virulence-related genes were identified (Table 2 and Fig. 2 (d)). The LM11T7 and LL13SP6 end regions gave best matches to the $a t t K$ and celB genes of a different strain of $A$. tumefaciens, respectively. The $a t t K$ gene has been reported to play a role in the attachment and virulence of $A$. tumefaciens C58 (Matthysse et al., 2000) while the celB gene of $A$. tumefaciens C58 plays a role in pathogenesis by producing cellulose fibrils, which bind the bacteria to host plant cells in the early stage of infection (Matthysse et al., 1995). Previous studies have identified $a t t K$ and $c e l B$ genes on the circular chromosome of A. tumefaciens C58 strain (Matthysse et al., 1995, 2000). Therefore, it will be of interest to conduct detailed studies to characterize these regions. The SK5SP6 endregion showed high homology to pyrroloquinoline-quinonedependent quinate dehydrogenase (qumA gene), which plays a role in quinate metabolism (Lee et al., 1999). The $a r o B$ gene homologue located in LM5Sp6 is another potential virulence-related gene, as this gene encodes the enzyme 3-dehydroquinate synthase, which catalyzes one of the early steps in the shikimate pathway (Bereswill et al., 1997). This pathway is present in many pathogenic organisms, and mutants of this gene have been shown to have attenuated pathogenicity.

Mobile gene homologues. Four insertion sequences which show high homology levels to A. tumefaciens were detected in the 1O11T7, 1O11SP6, 1I11SP6 and 1P10T7 end-regions (Table 2). As shown in Fig 2 (d), all four loci are situated at the left end region of the linear chromosome. Previous studies on a few linear replicons (Volf and Altenbuchner, 2000) have shown that genetically unstable sequences such as insertion sequences, transposons, antibiotic resistance/biosynthesis genes and genes responsible for secondary metabolic production tend to be closer to the telomeres. In contrast, housekeeping and rarely transferred and rearranged genes are located closer to the center of the linear chromosome (Volf and Altenbuchner, 2000).

Even though it is still premature to draw a definite conclusion, our results also show a similar pattern of distribution for the genes examined so far. The proposed insertion sequences are located towards the distal end (Table 2 and Fig. 2 (c)). A transport-system related gene (PA5167) and a housekeeping gene involved in citrate synthesis (HVAJ2075) at the T7 end of 2J24 clone were detected in this study, approximately $100 \mathrm{kbp}$ away from 
Table 3. Homologies of end-region sequences against Mesorhizobium loti ORFs

\begin{tabular}{lclcc}
\hline \hline Clone end (Accession No.) & Matching ORF & \multicolumn{1}{c}{ Functional category } & \%identity & Z score \\
\hline LN21SP6 (AB072632) & msl5187 & no similarity & 72 & 357 \\
2P4T7 (AB072637) & mll8314 & other category & 76 & 616 \\
1L9SP6 (AB072639) & mlr2977 & regulatory function & 61.8 & 517 \\
1L9T7 (AB072640) & mlr3359 & other category & 67 & 498 \\
2B3T7 (AB072644) & mlr3802 & regulatory function & 71.1 & 298 \\
2B3SP6 (AB072643) & mll3360 & conserved hypothetical protein & 63.5 & 380 \\
SE2T7 (AB072652) & mll5170 & other category & 60 & 226 \\
LM11SP6 (AB072662) & mlr3397 & other category & 68 & 501 \\
DG19T3 (AB072670) & mll5708 & conserved hypothetical protein & 73 & 421 \\
2E16T7 (AB072674) & mlr3477 & fatty acid phospholipid and sterol metabolism & 73 & 393 \\
BB1 T7(AB072682) & mlr3508 & central intermediary metabolism & 63.3 & 390 \\
LH2SP6 (AB072686) & mlr1663 & other category & 66.4 & 240 \\
LH9T7 (AB072695) & mlr3913 & conserved hypothetical protein & 71.7 & 475 \\
SI12SP6 (AB072694) & mll3968 & other category & \\
\hline
\end{tabular}

the end. Genes which are putative components of housekeeping/transport/virulence systems seem to be oriented centrally along the chromosome.

Homology with Mesorhizobium loti genome. Fourteen end-region sequences gave high homology only with Mesorhizobium loti sequences and their functions were deduced (Table 3, Kaneko et al., 2000). Another 17 endregions indicated in Table 2 also showed high homology with Mesorhizobium loti. In general, end-region sequencing identified a long region highly homologous to Mesorhizobium loti in the left region of the linear chromosome, in clones ranging from clones LN21 to $2 \mathrm{~B} 3$ and from clone SE2 to LH9 clone (Fig. 2 (c)). This indicates a common genomic relationship between the two organisms. Therefore, these data can be used as a guide to compare the existence of genes in those areas in the $M$. loti and A. tumefaciens genomes.

End-region sequencing analysis of the linkage library is a quick way to determine the genome organization of a specific region of a genome. A similar approach called "sequencing skimming" has been used as the basis for proposing the existence of genes in a few clones belonging to the linkage library of chromosome II of Rhodobacter sphaeroides 2.4.1(Mackenzie et al., 1999). Therefore, for the first time this study provides valuable genetic information about the left region of the linear chromosome of A. tumefaciens MAFF301001 and paves the way for detailed structural and functional genomic studies of it.

After the submission of this manuscript, the complete genome sequence data of a different strain, A. tumefaciens C58 appeared in DNA databases (NCBI genome accession No. NC003063 and DDBJ/EMBL/GenBank accession No.s AE008197-AE008383). Studies by Allardet-Servent et al. (1993) and Suzuki et al. (2001) clearly show some differences in the genomic make-up of the two Agrobacterium strains, MAFF301001 and C58. Casjens (1998) has explained the diverse and dynamic genome structures of microbial genomes and the possibility of having structural deviations even in genomes among strains of the same species. Therefore, these studies reinforce each other and provide more genetic details about the dynamic organization of genomes.

D. M. De C. is the recipient of a MONBUSHO scholarship by the Japanese Government. The authors are thankful to Dr. Ung-Jin Kim, California Institute of Technology, USA for providing the pFOS1 DNA.

\section{REFERENCES}

Allardet-Servent, A., Michaux-Charachon, S., Jumas - Bilak, E., Karayan, L., and Ramuz, M. (1993) Presence of one linear and one circular chromosome in the Agrobacterium tumefaciens C58 genome. J. Bacteriol. 175, 7869-7874.

Ames, G. F.-L. (1986) Bacterial periplasmic transport systems: structure, mechanism, and evolution. Annu. Rev. Biochem. 55, 397-425.

Bereswill, S., Fassbinder, F, Volzing, C., Haas, R., Reuter, K., Ficner, R., and Kist, M. (1997) Cloning and functional characterization of the gene encoding 3-dehydroquinate synthase (aroB) and tRNA-guanine transglycosylate (tgt) from Helicobacter pylori. Med. Microbiol. Immunol. (Berl). 186, $125-134$.

Casjens, S. (1998) The diverse and dynamic structure of bacterial genomes. Annu. Rev. Genet. 32, 339-377.

Clarke, L., and Carbon, J. (1976) A colony bank containing synthetic colE1 hybrid plasmids representative of the entire $E$. coli genome. Cell 9, 91-99.

Cooley, M. B., D' Souza, M. R., and Kado, C. I. (1991) The VirC and VirD operons of the Agrobacterium Ti plasmid are regulated by the ros chromosomal gene: Analysis of the cloned ros gene. J. Bacteriol. 173, 2608-2616.

Coulson, A., and Sulston, J. (1990) Genome mapping by restriction fingerprinting In: Genome analysis, a practical approach (eds.: K. E. Davies), pp 19-39. IRL Press, Oxford.

De Costa, D. M., Suzuki, K., and Yoshida, K. (2000) Toward structural and functional genomics of Agrobacterium tumefaciens: Linkage map of the left region of linear chromosome. Nucleic Acids Symp. Ser. 44, 97-98.

Douglas, C. J., Staneloni, R. J., Rubin, R. A., and Nester, E. W. (1985) Identification and genetic analysis of an Agrobacterium tumefaciens chromosomal virulence region. J. Bacteriol. 161, $850-860$. 
Hattori, Y., Suzuki, K., Uraji, M., Ohta, N., Katoh, A., and Yoshida, K. (1997) Genome structure of pTi-SAKURA (1): Strategy for DNA sequencing of a Japanese cherry-Ti plasmid. Nucleic Acids Symp. Ser. 37, 159-160.

Hattori, Y., Uraji, M., Suzuki, K., Ohta, N., Iwata, K., Kato, A., and Yoshida, K. (2000) Gene list on a plant tumor-inducing plasmid, pTi-SAKURA in Agrobacterium tumefaciens MAFF301001. DNA Res. 7, 371-380.

Hooykass, P. J. J., and Beijersbergen, A. G. M. (1994) The virulence system of Agrobacterium tumefaciens. Annu. Rev. Phytopathol, 32, 157-179.

Kaneko, T., Nakamura, Y., Sato, S., Asamizu, E., Kato, T., Sasamoto, S., Watanabe, A., Idesawa, K., Ishikwawa, A., Kawashima, K., Kimura, T., Kishida, Y., Kiyokawa, C., Kohara, M., Matsumoto, M., Matsuno, A., Mochizuki, Y., Nakayama, S., Nakazaki, N., Shimpo, S., Sugimoto, M., Takeuchi, C., Yamada, M., and Tabata, S. (2000) Complete genome structure of the nitrogen-fixing symbiotic bacterium Mesorhizobium loti (supplement). DNA Res. 7, 381-406.

Kim, U. J., Shizuya, H., de Jong, P. J., Birren, B., and Simon, M. I. (1992) Stable propagation of cosmid sized human DNA inserts in an $\mathrm{F}$ factor based vector. Nucl. Acids Res. 20, 1083-1085.

Kim, U. J., Birren, B. W., Slepak, T., Mancino, V., Boysen, C., Kang, H. L., Simon, M. I., and Shizuya, H. (1996) Construction and characterization of a human bacterial artificial chromosome library. Genomics 34, 213-218.

Klee, H., Horsch, R., and Rogers, S. (1987) Agrobacterium-mediated plant transformation and its further applications to plant biology. Annu. Rev. Plant Physiol. 38, 467-486.

Lee, Y. A., Lo, Y. C., and Yu, P. P. (1999) A gene involved in quinate metabolism is specific to one DNA homology group of Xanthomonas campestris. J. Appl. Microbiology. 87, 649658.

Mackenzie, C., Chidambaram, M., Choudhary, M., Nereng, K. S., Kaplan, S., and Weinstock, G. M. (1999) Sequence skimming of chromosome II of Rhodobacter sphaeroides 2.4.1 ${ }^{\mathrm{T}}$. In: Bacterial genomes: Physical structure and analysis (eds.: F. J. de Bruijn, J. R. Lupskim and G. M. Weinstock), pp. 541-551. Kluwer Academic Publishers, London.

Matthysse, A. G., White, S., and Lightfoot, R. (1995) Genes required for cellulose synthesis in Agrobacterium tumefaciens. J. Bacteriol. 177, 1069-1075.

Matthysse, A. G., Yarnall, H., Boles, S. B., and McMahan, S. (2000) A region of the Agrobacterium tumefaciens chromosome containing genes required for virulence and attachment to host cells. Biochim. Biophys. Acta 1490, 208-212.

Nishikawa, M., Suzuki, K., and Yoshida, K. (1992) DNA integration into recipient yeast chromosomes by trans-kingdom conjugation between Escherichia coli and Saccharomyces cerevisiae. Curr. Genet. 21, 101-108.

Nishikawa, M., and Yoshida, K. (1998) Trans-kingdom conjugation offers a powerful gene targeting tool in yeast. Genet. Anal. Biomol. Eng. 14, 65-73.
Rackwitz, H. R., Zehtner, G., Murialdo, H., Delius, H., Chai, J. H., Poustka, A., Frischauf, A., and Lehrach, H. (1985) Anaylsis of cosmids using linearization by phage lambda terminase. Gene 40, 259-266.

Redenbach, M., Kieser, H. M., Denapaite, D., Eichner, A., Cullum, J., Kinashi, H. and Hopwood, D. A. (1996) A set of ordered cosmids and a detailed genetic and physical map for $8 \mathrm{Mb}$ Streptomyces coelicolor A3(2) chromosome. Mol. Micorbiol. 21, 77-96.

Sambrook, J., Fritsch, E. F., and Maniatis, T. (1989) Molecular cloning: A Laboratory Manual. 2nd Ed., Cold Spring Harbor Laboratory, Cold Spring Harbor, New York.

Sawasaki, Y., Inomata, K., and Yoshida, K. (1996) Trans-kingdom conjugation between Agrobacterium tumefaciens and Saccharomyces cerevisiae, a bacterium and a yeast. Plant Cell Physiol. 37, 103-106.

Sheng, J., and Citovsky, V. (1996) Agrobacterium-plant cell DNA transport: Have virulence proteins, will travel. Plant Cell 8, 1699-1710.

Suzuki, K., Ohta, N., Hattori, Y., Uraji, M., Katoh, A., and Yoshida, K. (1998) Novel structural difference between nopaline- and octopine-type trbJ genes: Construction of genetic and physical map and sequencing of trb/traI and rep gene clusters of a new Ti plasmid pTi-SAKURA. Biochim. Biophys. Acta 1396, $1-7$.

Suzuki, K., Hattori, Y., Uraji, M., Ohta, N., Iwata, K., Murata, K., Kato, A., and Yoshida, K. (2000) Complete nucleotide sequence of a plant tumor-inducing Ti plasmid. Gene $\mathbf{2 4 2}$, 331-336.

Suzuki, K., Iwata, K., and Yoshida, K. (2001) Genome analysis of Agrobacterium tumefaciens: Construction of physical map of linear and circular chromosomal DNAs, determination of copy number ratio and mapping of chromosomal virulence genes. DNA Res. 8, 141-152.

Vimont, S., and Berche, P. (2000) NhaA, an $\mathrm{Na}(+) / \mathrm{H}(+)$ antiporter involved in environmental survival of Vibrio cholerae. J. Bacteriol. 182, 2937-2944.

Volff, J-N., and Altenbuchner, J. (2000) A new beginning with new ends: linearisation of circular chromosomes during bacterial evolution. FEMS Microbiol. Lett. 186, 143-150.

West, S. C. (1997) Processing of recombination intermediates by the RuvABC proteins. Annu. Rev. Genet. 31, 213-244.

Winans, S. C. (1992) Two-way chemical signaling in Agrobacterium-plant interactions. Microbiol. Rev. 56, 12-31.

Wolfgang, J. P., Poulet, S., Eiglmeier, K., Pascopella, L., Balasubramanian, V., Heym, B., Bergh, S., Bloom, B. R., Jacobs Jr., W. R. and Cole, S. T. (1996) An integrated map of the genome of the tubercle bacillus, Mycobacterium tuberculosis H37Rv, and comparison with Mycobacterium leprae. Proc. Natl. Acad. Sci. USA. 93, 3132-3137.

Zambryski, P. C. (1992) Chronicles from the Agrobacterium-plant cell DNA transfer story. Annu. Rev. Plant Physiol. Plant Mol. Biol. 43, 465-490. 